\title{
IL PROGETTO DELLA PSICOLOGIA DELLE MENTI ASSOCIATE
}

\author{
MARIACHIARA FUGAZZA (*)
}

SunTo. - La recente edizione delle letture tenute da Carlo Cattaneo all'Istituto Lombardo dal 1859 al 1866 è uno strumento utile per accostarsi a questo lavoro conclusivo, uno dei più significativi della produzione teorica dell'autore. Dall'edizione emergono varie notizie relative al progetto delle conferenze e ai legami con altri scritti di Cattaneo, in particolare con quanto da lui prodotto come insegnante di filosofia al Liceo di Lugano. Il tentativo di dare nuovo fondamento alla filosofia e di mettere a punto un metodo di comprensione del divenire delle società è uno dei motivi centrali nell'esperienza intellettuale dello scrittore milanese, che tuttavia concepisce una "psicologia delle menti associate" come necessario complemento della psicologia individuale soprattutto nella seconda parte della sua vita. L'idea di fondo è che ogni attività umana, e in particolare la conoscenza scientifica, dipendono dalla natura collettiva del pensiero, base del progresso dell'umanità da cui nessun popolo è escluso a priori. La riflessione più tarda di Cattaneo appare un'originale filosofia dell'intelligenza: nelle letture all'Istituto egli si mostra fiducioso nel progresso, ma consapevole del suo carattere non garantito; ammiratore della scienza, ma contrario a ogni interpretazione deterministica dello sviluppo del pensiero e delle culture.

$$
* * *
$$

ABSTRACT. - The new edition of the lectures delivered by Carlo Cattaneo at the Istituto Lombardo from 1859 to 1866 is a useful tool for understanding one of his last and most important theoretical works. It offers information on the project of the lectures and on their connection with other Cattaneo's works and in particular with his activity of teacher of Philosophy at the Lugano Liceo. The effort to give new basis to philosophy and to find a method for a sociological approach to history has always been a focus of Cattaneo's intellectual experience. Nevertheless he conceives his "psicologia delle menti associate" as a necessary complement of individual psychology especially in the second part of his life. His basic idea is that any human activity and above all scientific researches depend on the universal nature of thought, which is the source of human

(*) Comitato italo-svizzero per la pubblicazione delle Opere di Carlo Cattaneo, Milano, Italia. E-mail: mc.fugazza@gmail.com 
progress and involves every people. So Cattaneo's last work seems like a personal philosophy of intelligence. In the lectures at the Istituto Lombardo he appears as confident in progress, but at the same time is aware that a decline is always possible. He admires Sciences, but he rejects any deterministic explanation of the development of human thought and cultures.

Il fatto di non considerare la filosofia un proprio territorio d'elezione e di non coltivare sistematicamente questa disciplina non impedì a Cattaneo di dare contributi originali, accostandosi a temi filosofici ${ }^{1}$ in fasi diverse della vita e combattendovi importanti battaglie di principio. $\mathrm{Al}$ pari di altri scritti della sua produzione, in non pochi casi i suoi testi filosofici furono inizialmente abbozzati come appunti, destinati solo in parte a convergere in saggi predisposti per la stampa. Legati a esigenze non di rado divulgative o didattiche, nell'arco dell'esistenza dell'autore essi videro talvolta la luce in forma compendiata, e solo dopo la sua morte furono pubblicati da coloro che assunsero l'incarico di farne conoscere i contenuti, ricavando i materiali all'interno di quel grande patrimonio documentario costituito dall'archivio. Sottolineare il carattere degli scritti teorici prodotti da Cattaneo specialmente nell'ultima fase della vita, far presente che accanto a quanto conosciuto esistono schemi e annotazioni frammentarie, è importante per intendere il metodo di questo studioso, il quale per successive tappe identificò le linee del suo discorso, con l'ambizione non di aggiungersi al panorama affollato degli ontologi e di quelli che definiva «cercatori del vero primo» ${ }^{2}$, ma di dare suggerimenti finalizzati al rinnovamento del sapere di cui voleva farsi portavoce.

Alla luce delle caratteristiche del Cattaneo filosofo, si comprende il significato dell'iniziativa promossa recentemente dall'Istituto

1 Uno dei rimandi tuttora essenziali per l'analisi di questo versante della produzione cattaneana è il lavoro svolto da Norberto Bobbio per la cura di C. Cattaneo, Scritti filosofici, 3 voll., Firenze, Le Monnier, 1960 (d'ora in avanti SF), e nei saggi confluiti in N. Bobbio Una filosofia militante. Studi su Carlo Cattaneo, Torino, Einaudi, 1971.

2 SF, I, 253. L'espressione è tratta dalla prefazione al volume VI del Politecnico dove, con toni scopertamente ironici, Cattaneo parla in realtà di «pescatori del vero primo», espressione poi corretta nella versione inserita in Alcuni scritti del dottor Carlo Cattaneo, III, Milano, Borroni e Scotti, 1847 e da qui riportata da Bobbio nella raccolta del 1960. 
Lombardo, e cioè un'edizione delle letture sulla Psicologia delle menti associate tenute all'Istituto tra il 1859 e il 1866, già note attraverso precedenti pubblicazioni e ora per la prima volta proposte nelle loro differenti versioni ${ }^{3}$. Dopo la presentazione di Enrico Decleva e un saggio di Carlo G. Lacaita, che ricostruisce la trama complessiva dell'itinerario cattaneano, il volume contiene a cura di Barbara Boneschi gli estratti delle lezioni inseriti negli Atti e nei Rendiconti dell'Istituto, le stesure che vennero all'epoca diffuse da alcuni periodici, quelle più ampie ricavate dai manoscritti e già apparse in varie sedi e infine schemi e abbozzi pure esistenti nelle carte. L'insieme consente di accostarsi in modo approfondito a quest'opera tarda dell'autore milanese, che come altre dovute alla sua penna è stata oggetto nel tempo di diverse valutazioni critiche.

$\mathrm{Al}$ centro delle conferenze è lo sforzo di concorrere alla necessaria e a parere dello scrittore ineludibile convergenza tra scienze della natura e mondo umano, attraverso la valorizzazione dell'origine sociale del pensiero, e del pensiero scientifico in particolare, quale patrimonio comune prodotto dall'umanità nel corso delle generazioni. E poiché tale visione risulta elaborata da Cattaneo con una fondamentale coerenza tra i principali motivi entro cui venne svolgendosi la sua riflessione, pur avendo come obiettivo l'analisi delle lezioni che ebbero luogo dal 1859 al 1866, il presente contributo cercherà di rintracciare nei lavori precedenti le radici della sua produzione più tarda, e di quella filosofia dell'intelligenza che si rivela esserne il nucleo più originale.

\section{GLI ANTECEDENTI MILANESI}

Alcune indispensabili premesse. È ben noto che qualunque analisi delle concezioni di Cattaneo non può prescindere dal richiamo alle esperienze condotte alla scuola di Gian Domenico Romagnosi, dato che proprio nella cerchia del grande giurista maturò nella Milano della Restaurazione il disegno di un sapere sull'individuo associato e

3 C. Cattaneo, Psicologia delle menti associate. Le letture di Carlo Cattaneo all'Istituto Lombardo di Scienze e Lettere. Edizione critica a cura di B. Boneschi. Presentazione di E. Decleva. Saggio di C.G. Lacaita, Istituto Lombardo di Scienze e Lettere, Milano, 2016 (d'ora in avanti C. Cattaneo, Psicologia). 
sull'incivilimento, volto all'obiettivo di indagare le leggi del divenire storico, destinato a influire profondamente sulla formazione del giovane discepolo ${ }^{4}$. Interessato inizialmente soprattutto a ricerche di economia e di linguistica, sulla scorta degli insegnamenti del maestro e a commento di alcuni scritti di quest'ultimo, Cattaneo cominciò infatti ad addentrarsi in questioni teoriche più impegnative, e in quest'ambito un ruolo primario svolse come si sa la riconsiderazione dell'eredità di Giambattista Vico. In un notissimo saggio del $1839^{5}$, discutendo il lascito del filosofo napoletano, Cattaneo richiamò con ricchezza di argomenti alcuni dei temi destinati a diventare decisivi nella sua esperienza intellettuale. Si trattava, contro una filosofia dell'uomo astrattamente considerata, della affermata impossibilità di «scrutare l'essenza» dello spirito umano e della necessità di conoscerlo nelle sue manifestazioni, perché da tale terreno «tutto istorico ed esperimentale» sarebbe sorta «l'intera cognizione dell'uomo, la quale indarno si cerca nelle latebre della solitaria coscienza» ${ }^{6}$. Era questa la scienza fondata da Vico, l'ideologia sociale ripetutamente evocata nei successivi lavori cattaneani.

Indicato come iniziatore di uno studio non più rivolto alla genesi

4 In proposito cfr. R. Ghiringhelli, La filosofia civile di Carlo Cattaneo ovvero Romagnosi e Cattaneo, in Carlo Cattaneo: i temi e le sfide, a cura di A. Colombo, F. Della Peruta e C.G. Lacaita, Milano, G. Casagrande, 2004, 317-323. Sul tema dell'incivilimento e sull'influsso esercitato in particolare dall'ultimo Romagnosi, M. Martirano, $L a$ filosofia civile in alcuni momenti del pensiero democratico risorgimentale, in Momenti della filosofia civile italiana, a cura di G. Cacciatore e M. Martirano, Napoli, La città del Sole, 2008, 147-200.

5 C. Cattaneo, Vico et l'Italie, pubblicato in Politecnico, 1839, II, fasc. IX, 251 286; dalla versione di Alcuni scritti e con il titolo Su la «Scienza Nuova» di Vico, in SF, I, 95-138.

6 Ivi, 103. Il concetto ricorre con alcune varianti anche in una stesura preparatoria del saggio del 1839: «Ora le fasi sociali e le produzioni intellettuali in cui si svolgono i poteri dell'animo umano sono disegnate nella istoria, e nella etnografia, nella letteratura, nelle arti, nelle leggi, nelle lingue, in ogni sorta di umane instituzioni. Da questo campo vastissimo tutto istorico ed esperimentale sorge la vera cognizione dell'uomo, lo studio dell'individuo per mezzo dell'umanità, l'ideologia [corr. di: «la psicologia»] sociale. È questa la scienza fondata da Vico». Cfr. M. Fugazza, Filosofia e scienze umane: intorno ad alcuni autografi di Cattaneo, in Cattaneo, Milano e la Lombardia, Incontro di studio n. 28, Milano, 29-30 novembre 2001, Milano, Istituto LombardoAccademia di Scienze e Lettere, 2005, 221. 
delle idee dell'individuo, ma ai principi ispiratori dell'esistenza delle collettività nel tempo, il filosofo napoletano sarebbe stato anche in seguito per Cattaneo un riferimento costante, pur con una significativa inversione di prospettive. Se cioè Vico aveva cercato «nell'animo umano il principio istorico», procedendo all'opposto e cioè «invertendo l'andamento» si sarebbe potuto «con maggior sicurezza inferire dai fatti innumerevoli dell'istoria le forze e le inclinazioni dell'uomo interiore», ritrovandole «nelle vicende delle arti e delle lettere e delle religioni e dei commerci e delle industrie, e infine della scienza, ora stagnante in labirinti contemplativi, ora con subito impeto innovatrice e feconda» ${ }^{7}$. Sulla scia della lettura condotta da Romagnosi, accanto al riconoscimento delle grandi intuizioni vichiane non mancavano dunque motivi di distinzione, che si compendiavano in un diverso modo di intendere il divenire storico e la varietà come segno caratteristico e peculiare della vicenda delle comunità sulla terra. Un progresso inimmaginabile ai tempi di Vico emergeva ormai evidente dal corso delle cose, dai fatti prodotti da un «secolo di meraviglie» ${ }^{8}$. La varietà era il risultato di una cognizione straordinariamente ampliata, rispetto alle esperienze dell'autore della Scienza nuova, dei volti delle civiltà, e quindi «dei monumenti, delle croniche, delle religioni, delle sètte, delle filosofie, delle arti, delle leggi, dei governi, delle legende, delle letterature, delle lingue, e perfino dei dialetti»?.

Negli anni prequarantotteschi tali concezioni ebbero da parte di Cattaneo una traduzione "militante", coincidente con l'esperienza del Politecnico, il «repertorio di studj applicati alla prosperità e coltura sociale», uscito dal 1839 al 1844 e divenuto l'organo di diffusione del suo programma. Alla base della rivista lo studioso milanese pose una visione che, a somiglianza delle scienze della natura, avrebbe collocato su un cammino rinnovato anche il sapere sull'uomo e sulla società. Nello sforzo di convergenza tra le discipline, in vista della fondazione di un armonico sistema delle conoscenze, il giornale includeva pertanto in un unico quadro di riferimento le arti «figlie delle scienze matematiche e fisiche» e l'«immenso apparato dell'arte sociale»; e ancora le «arti mentali», in cui l'uomo può «farsi studio della parte intima di sè stesso»

7 SF, I, 251.

8 Ivi, 124.

9 Ibidem. 
e le «arti belle» ${ }^{10}$. Parallelamente, nelle prefazioni dovute alla penna del direttore, poste in apertura dei volumi corrispondenti alle annate del periodico, erano ripresi e discussi i presupposti metodologici dell'organizzazione degli argomenti trattati.

In questi scritti, in una combinazione di spunti teorici ed esortazioni "civili", la visione cattaneana di una fondamentale e concorde unità del pensiero trovò alcune delle sintesi più felici, quale è quella che si legge nella prefazione al volume II: «I popoli debbono farsi continuo specchio fra loro, perché li interessi della civiltà sono solidari e communi; perché la scienza è una, l'arte è una, la gloria è una. La nazione delli uomini studiosi è una sola: è la nazione d'Omero e di Dante, di Galileo e di Bacone, di Volta e di Linneo, e di tutti quelli che seguono i loro esempi immortali; è la nazione delle intelligenze, che abita tutti i climi e parla tutte le lingue» ${ }^{11}$. E ancora, «il dover nostro è d'accrescere nella patria che abitiamo, colla lingua che parliamo, e colle felici attitudini naturali della nostra stirpe il dominio delle intelligenze, e detrarre quanto si può alla rozzezza originaria che forma dappertutto il fondo delle nazioni ${ }^{12}$. A dimostrazione di una consapevolezza sempre più salda, nella prefazione al volume del 1843, a proposito della «industria scientifica, disseminata vastamente fra i popoli pensanti», si osservava: «Ogni scienza è un vasto pensiero. Le singole scienze, o diremo, i singoli pensieri, divisi nella loro partenza, indipendenti nelle loro vie, devono far prova della veracità loro, convergendo finalmente ad un punto, ove si fondono in un riassunto commune e concorde, il quale potrebbe chiamarsi per eccellenza il pensiero, il pensiero del genere umano» ${ }^{13}$. Nella medesima prefazione, al fine di indagare non solo il barlume di coscienza dell'individuo alla nascita, ma le infinite varietà rintracciabili nella storia dei popoli, dei loro linguaggi e delle loro civiltà, la proposta era di prendere «per argomento del pensiero il pensiero stesso» ${ }^{14}$.

10 Per una panoramica della vita e dei collaboratori della testata, cfr. «Il Politecnico» di Carlo Cattaneo. La vicenda editoriale, i collaboratori, gli indici, a cura di C.G. Lacaita, R. Gobbo, E.R. Laforgia, M. Priano, Milano-Lugano, G. Casagrande, 2005.

11 SF, I, 233.

12 Ivi, 234.

13 Ivi, 246-247.

14 Ivi, 250. 
Come conseguenza delle riflessioni che era andato maturando attraverso l'esperienza del Politecnico, quando, in seguito alla sospensione dello stesso, Cattaneo ebbe nel 1846 l'idea di radunare in una nuova pubblicazione i suoi saggi affidati alle pagine del mensile ${ }^{15}$, dopo un primo volume dedicato alla letteratura e alla linguistica e un secondo alla storia, egli ne progettò un terzo che avrebbe dovuto rappresentare i due rami della filosofia civile e naturale, in vista di una nuova fondazione della disciplina. Nella prima sezione, sotto il titolo di filosofia civile, vennero così riproposti testi teorici già usciti nella rivista, inclusi il saggio su Vico e frammenti delle sette prefazioni. Il tutto come contributo a un sapere sull'uomo e sulla società che concorresse a distaccare la scienza del pensiero dal «vetusto romanzo della prima idea», avviando anche le dottrine morali sull'«avventuroso cammino» già intrapreso dalle scienze fisiche.

Tra i lavori inseriti nella raccolta, oltre a quelli citati merita particolare attenzione un articolo pubblicato nel 1844 all'epilogo della prima serie del giornale, a commento di un'opera di Giuseppe Ferrari edita in Francia ${ }^{16}$. Adottando la forma consueta della lunga recensione, Cattaneo tornava nel testo su alcuni temi ben noti, quali la critica a ogni filosofia lontana dall'esperienza, la presa di distanze dalla metafisica e dall'ontologia, il riferimento agli insegnamenti della Scienza nuova, pur con distinzioni rispetto a un pensiero considerato troppo legato alla ricerca di «ripetizioni e similarità», dalle quali, come era letteralmente affermato, era ormai tempo di prescindere per «delibare una volta le altre infinite varietà dell'istorica descrizione» ${ }^{17}$.

Riprendendo le suggestioni ferrariane, una parte consistente del

15 Nella già citata raccolta Alcuni scritti del dottor Carlo Cattaneo, pubblicata a Milano da Borroni e Scotti nel 1846-1847.

16 Essai sur le principe et les limites de la philosophie de l'bistoire, Paris, Joubert, 1843. Come provano le bozze conservate in Raccolte Storiche del Comune di Milano, Archivio Carlo Cattaneo, cart. 17, pl. III, n. 4, per aggirare i divieti della censura la recensione di Cattaneo dovette essere modificata dall'autore, sopprimendo i richiami diretti all'opera ferrariana. Con il titolo Considerazioni sul principio della filosofia fu pubblicata in Politecnico, 1844, VII, fasc. XXXIX, 292-313. Con lo stesso titolo, ma con l'aggiunta di alcune note e ripristinando i riferimenti al volume di Ferrari, essa venne ristampata in Alcuni scritti, III. cit., 39-60, e da questa versione in SF, I, 153-170.

17 SF, I, 153 e 155. 
discorso verteva intorno al concetto di sistema. A quello stadio di elaborazione, la trasformazione in sistema era ancora collegata con la fase di ripiegamento di ogni civiltà in declino, incapace di accogliere in sé la pluralità di princìpi che era invece propria delle società dinamiche e progressive. Rispetto a tale visione, taluni punti del saggio prefiguravano tuttavia una spiegazione già più articolata e complessa dell'avvicendamento storico: «Le nazioni civili racchiudono in sé vari principî, ognuno dei quali aspira a invadere tutto lo stato, e modellarlo in esclusivo sistema. Ma prima che l'opera sia compiuta, nuovi principî si svolgono in modo imprevisto, e dirigono verso altra parte la corrente delli interessi e delle opinioni» ${ }^{18}$. La concezione delle civiltà come sistemi organizzati, destinati a venir meno attraverso la loro integrale sostituzione, pareva di conseguenza da integrare e correggere: «Non sembra adunque potersi consentir facilmente che vi sia una legge fondamentale nelli umani concorsi per la quale le idee non possano esistere senza ordinarsi in sistema: che quindi ogni civiltà formi necessariamente sistema, il quale non possa mai cadere se non per sostituzione d'altro sistema. I principî civili, a noi pare, sono come le quantità, le quali per minime aggiunte o minime detrazioni mutano assolutamente il punto d'equilibrio» ${ }^{19}$. In merito ai meccanismi interni alla dinamica delle collettività, Cattaneo indicava come si vede un ampio territorio di indagini ${ }^{20}$. Sarebbero tuttavia passati vari anni perché queste suggestioni potessero essere sviluppate in una successiva stagione della sua produzione.

\section{Da Milano a Lugano}

A causa degli avvenimenti del 1848, gli interessi dello scrittore presero infatti per qualche tempo tutt'altro indirizzo, dato che la prima parte dell'esilio in territorio elvetico fu interamente occupata

18 Ivi, 157.

19 Ivi, 158-159.

20 Riassunto in due «asserti» presentati quali capisaldi di una prospettiva da approfondire e sviluppare, e cioè: «Quanto più civile è un popolo tanto più numerosi sono i principî che nel suo seno racchiude» e «L'istoria è l'eterno contrasto dei diversi principî che tendono ad assorbire e uniformare la nazione», ivi, 157-158 e nota. 
dagli echi dei recenti fatti. Da "archivista" e storico della rivoluzione, collaborando intensamente soprattutto con la Tipografia Elvetica di Capolago, egli varò una serie di impegnativi progetti editoriali finalizzati alla documentazione degli eventi trascorsi. Parallelamente, con l'intensificarsi della partecipazione alla vicende del Canton Ticino, divenuto per lui una seconda patria, iniziò ad assumere compiti di crescente responsabilità affidatigli dalle autorità cantonali ${ }^{21}$. Tra essi in particolare l'incarico, portato a termine nel 1852, di disegnare il quadro della riforma degli studi del Canton Ticino, che aveva un immediato antecedente nel ruolo svolto nell'ambito di una commissione istituita nell'Istituto Lombardo nei mesi che avevano preceduto l'insurrezione ${ }^{22}$. Prima e più ancora che come docente, egli fu dunque protagonista del piano generale di riorganizzazione dell'istruzione superiore del Cantone, che suscitò il plauso delle autorità e insieme la vivace opposizione degli ambienti conservatori. Nel meditare i presupposti di una nuova modulazione dei saperi, l'ex capo del milanese Consiglio di guerra si trovò così ad attingere al complesso delle convinzioni teoriche elaborate nella Milano prequarantottesca.

La riforma da lui delineata prese in considerazione aspetti diversi convergenti in un disegno, che non è possibile considerare nel suo insieme in questa sede. Ai fini della presente ricostruzione è interessante osservare che, in merito al nucleo centrale dell'insegnamento filosofico, in polemica con «le solite, insolubili controversie di metafisica e di psicologia», nel progetto presentato lo scrittore sintetizzò in questi termini le sue indicazioni: «Evitando l'astratta contemplazione d'un solitario astratto individuo, vorrebbesi considerare li uomini, come veramente surgono nel consorzio dei loro simili, ed esporre istoricamente come le loro facultà mentali e morali vanno assiduamente modificandosi nei vari stati di selvatichezza, di barbarie, di civiltà, di decadimento, e nel continuo conflitto delle tradizioni e del progresso, della credulità e della ragione, del privilegio e dell'eguaglianza, della servitù e della

21 Per una panoramica d'insieme dell'attività del Cattaneo ticinese, cfr. C. Moos, «L'altro» Risorgimento. L'ultimo Cattaneo tra Italia e Svizzera, Milano, FrancoAngeli, 1992.

22 Cfr. C. Cattaneo, Sull'ulteriore sviluppo del pubblico insegnamento in Lombardia, in Id., Tutte le opere, a cura di L. Ambrosoli, Milano, Mondadori, 1967, IV, 3-54 e note relative. 
libertà $»^{23}$. Solo dopo aver esaminato la varietà dei sistemi sociali e delle loro trasformazioni nel tempo, si sarebbe potuto accedere alle «leggi supreme dell'intelligenza», una volta trattate «la dottrina del progresso», quella «dei sistemi sociali, delle innovazioni e rivoluzioni», passando poi «all'influenza reciproca delle genti, delle religioni, delle idee, delle scoperte, dei sofismi, degli errori, delle scienze pure e delle scienze applicate, dei metodi logici ed esperimentali», arrivando «all'ordine umanitario e universale», e ritornando in tal modo «all'arcano e sublime punto d'onde si presero le mosse».

Coerentemente con simili vaste linee programmatiche, pronunciando nel 1852 la Prolusione $e^{24}$ in occasione della solenne inaugurazione del Liceo, Cattaneo prefigurò un corso filosofico nel quale la dimensione sociale e storica era affermata con grande evidenza, come provano molti passaggi del testo: «È cosa strana, ma li scrittori amarono sempre indagar l'origine delle idee piuttosto nell'uomo individuo, che nell'uomo associato. Anzi discesero quasi sempre all'intelletto dell'infante, supponendolo solitario e derelitto al cospetto del mondo esteriore, e imaginando che colle mere sue forze dovesse fabricarsi ad una ad una tutte le idee, come se l'umanità dovesse per ogni individuo ricominciar sempre da' suoi primordi selvaggi ${ }^{25}$. Constatato che sin dal principio della vita l'essere umano trae le sue idee non da se stesso, ma da una madre inserita in una comunità, così argomentava: «Ogni idea dell'infante non è adunque l'opera d'una mente solitaria, ma di più menti associate $\rangle^{26}$. Al pari delle discipline che avevano messo a punto un metodo efficace, la filosofia, procedendo dal noto all'ignoto, avrebbe dovuto quindi considerare l'«intelletto individuo» non dall'inizio, ma da ultimo, avendo affrontato uno studio delle lingue, delle letterature, delle mitologie, delle leggi, delle scienze e delle «altre grandi elaborazioni delle menti adulte e associate» ${ }^{27}$.

23 Il testo del progetto di riforma, apparso unitamente a quello di Giovanni Cantoni nel Supplimento straordinario al Foglio officiale delle pubblicazioni e degli annunzii nel Cantone Ticino, Bellinzona, 21 aprile 1852, è riportato in C. Cattaneo, Scritti politici, a cura di M. Boneschi, Firenze, Le Monnier, 1965, III, 67-97. Ivi, 80-82 la parte relativa all'insegnamento della filosofia, da cui sono tratte le citazioni.

24 Stampata a Capolago, presso la Tipografia Elvetica, nel novembre 1852, e riportata in $S F$, II, 10-26.

25 Ivi, 13-14.

26 Ivi, 16.

27 Ivi, 17. 
Successivamente alla stesura del piano di riforma, l'insegnamento della materia fu assunto da Cattaneo non senza qualche iniziale perplessità, per la condizione di docente di una disciplina che non considerava suo specifico ambito di competenza ${ }^{28}$, ma poi, come ebbe a confidare a distanza di qualche anno ad Enrico Cernuschi, l'esperienza gli rivelò inedite prospettive ${ }^{29}$. Riguardo alle intenzioni dichiarate, la necessità di identificare $\mathrm{i}$ capitoli da trattare implicò in verità alcune modifiche ${ }^{30}$, orientate a includere non solo la filosofia civile, ma anche riferimenti alle scienze naturali, che portarono alla divisione dei programmi in cinque sezioni: cosmologia (sintesi di conoscenze sull'universo fisico e sul posto dell'uomo nel medesimo, che attirò le critiche più accese da parte di conservatori e clericali ostili a tutto lo spirito della riforma), e quindi psicologia, ideologia, logica e diritto, inteso quale principio di morale e di legislazione, e cioè, come si vede, branche solo in parte ordinariamente previste nella consueta organizzazione degli studi.

L'eco della riforma disegnata con la Prolusione e poi con l'avvio delle lezioni superò ben presto i confini del piccolo Cantone. Da Genova Carlo Pisacane, che aveva potuto leggere il testo inaugurale grazie a Mauro Macchi, ne parlò ad esempio con compiacimento, elogiando l'autore per aver «iniziato sul versante delle Alpi» la scienza, che un giorno auspicabilmente si sarebbe irraggiata anche in Italia, dato che non vi era questione politica o religiosa che non sarebbe entrata nel vasto campo ora dischiuso ${ }^{31}$. Altre testimonianze provano l'importanza

28 Cfr. la lettera alla Tipografia Elvetica del 3 ottobre 1852, in Carteggi di Carlo Cattaneo, serie I: Lettere di Cattaneo, III, 1852-1856, a cura di M. Cancarini Petroboni e M. Fugazza, Firenze-Bellinzona, Le Monnier-Casagrande, 2010, 45.

29 «Io son contento d'essere stato condotto dalle circostanze a rifare studj dai quali aveva ritorto l'animo; e ho trovato che vi è ancora terra nuova», lettera a E. Cernuschi del 17 maggio 1855, ivi, 222.

30 Cfr. i Programmi del corso di filosofia in SF, II, 3-9, pubblicati in apertura delle lezioni. Queste ultime, riportate in SF, II e III, furono ricavate da Bobbio in gran parte (salvo la Prolusione e tre capitoli delle lezioni di cosmologia) dai manoscritti dell'Archivio Carlo Cattaneo presso le Raccolte Storiche del Comune di Milano. Per l'analisi delle stesse nell'ambito dell'opera cattaneana, cfr. Id., Una filosofia militante, cit., 139-181.

31 Le affermazioni sono tratte dalla lettera a Cattaneo, che si trova sotto la data del 17 gennaio 1853 in C. Pisacane, Epistolario, a cura di A. Romano, Milano-GenovaRoma-Napoli, Soc. anonima Dante Alighieri, 1937, 155-157. 
attribuita negli ambienti dell'emigrazione patriottica a quell'incarico didattico, come pure per opposti motivi nei circuiti dell'opposizione conservatrice, che a lungo polemizzò con le linee enunciate per il nuovo sistema di istruzione ${ }^{32}$. A distanza di anni, nel 1860, a dimostrazione della rilevanza del suo discorso programmatico, lo stesso Cattaneo avrebbe ripubblicato la Prolusione nella nuova serie del Politecnico ${ }^{33}$.

Tornando ai corsi, le lezioni di psicologia, ricalcando in gran parte impostazioni manualistiche, trattavano dopo una introduzione generale 23 argomenti che andavano dall'istinto e dalla sensazione fino alla volontà. Come provano gli appunti esaminati da Bobbio, l'autore rivide e modificò più volte questa, come le altre parti del suo insegnamento, dal momento che sui manoscritti stratificati di correzioni sono apposte date successive: $1857,1859,1861$ e $1865^{34}$. Una analoga parentela con motivi affrontati in momenti differenti della sua esperienza intellettuale si può riconoscere in relazione all'ideologia, che nelle sue articolazioni (ideologia propria, linguistica o segni delle idee, e soprattutto i 17 capitoli di ideologia delle genti) offriva un embrionale sommario di storia delle civiltà ${ }^{35}$. E non sorprende che dopo l'ideologia dell'individuo e la linguistica quale parte dell'ideologia, come padre della parte terza, cioè della «istoria delle idee nel seno della società», fosse diffusamente evocato il nome di Giambattista Vico ${ }^{36}$, il pensatore che come si è detto era stato in ambito milanese e per la cerchia di Romagnosi in specie un punto di riferimento essenziale e, con Bacone e Locke, era entrato a pieno titolo nel ristretto pantheon filosofico cattaneano.

32 Ancora nel 1858, l'azione svolta nell'ambito del sistema scolastico cantonale e particolarmente il testo inaugurale del 1852 furono all'origine degli attacchi assai violenti sferrati all'esule dalle pagine del Credente cattolico.

33 Prolusione a un corso di filosofia nel Liceo ticinese del Dr. Carlo Cattaneo, Politecnico, 1860, VIII, fasc. XLIII, 60-71. Il testo era accompagnato dalla seguente nota: «Questa prolusione aperse il corso di filosofia nel nuovo Liceo del Ticino in novembre 1852. Non potè allora aver libera circolazione; e ora, in mancanza d'altro programma, potrà dare impulso a nuovo studj. Il vasto e vario argomento attende la perseverante opera di molti».

34 Cfr. quanto si legge nella Nota di Bobbio in SF, I, LXIII.

35 Sul pensiero di Cattaneo in relazione allo studio delle forme culturali, cfr. le considerazioni proposte da C.T. Altan, L'ideologia delle genti, in Carlo Cattaneo e il Politecnico, a cura di A. Colombo e C. Montaleone, Milano, Franco Angeli, 1993, 257-265.

36 Cfr. la sintesi del pensiero vichiano contenuta nelle lezioni di Ideologia, in SF, II, 307-310. 


\section{IL “CANTIERE” DELLA PSICOLOGIA DELLE MENTI ASSOCIATE}

Quanto resta tra le carte prova che, nell'ambito del lavoro continuativo svolto per il Liceo, nacque con ogni evidenza in Cattaneo il progetto di dedicarsi a testi in cui ampliare alcune intuizioni che, dalle premesse più lontane ai recenti compiti legati ai nuovi scopi didattici, egli considerava maggiormente meritevoli di approfondimenti. Da qui il primo nucleo di osservazioni da cui sarebbe scaturito il contenuto delle conferenze all'Istituto Lombardo.

Dopo le anticipazioni della Prolusione, le lezioni luganesi di psicologia, pur non diffondendosi sulla natura sociale del pensiero, contenevano di nuovo accenni al tema, come dimostra il seguente passo, tratto dal capitolo I del corso: «Le facultà, sviluppandosi a poco a poco, si elevano dall'instinto e dai gradi infimi dell'intelligenza fino al ragionamento scientifico e inventivo, ch'è la meta ultima a cui la mente può pervenire. Questa aspirazione costituisce l'idea del progresso. Ma ciò non avviene nell'uomo isolato, né in una società di pochi uomini, e nemmeno in una sola nazione; ma nella successione continua dei tempi, in seno di molte nazioni, nell'umanità intera. L'edificio della scienza è l'opera, non della mente solitaria, ma delle menti associate»> ${ }^{37}$.

Erano questi i motivi che Cattaneo intendeva riprendere e intorno a cui voleva lavorare. Anche i manoscritti presentati nell'edizione che ha recentemente visto la luce provano che «Psicologia delle menti associate» fu un'espressione intorno a cui egli iniziò a soffermarsi più sistematicamente proprio in quegli anni Cinquanta. «Psicologia seconda o istoria delle menti associate», come necessario complemento della psicologia della mente solitaria, è una dicitura presente ad esempio in uno schema esistente in archivio ${ }^{38}$. E ancora, il sopratitolo Delle menti associate o psicologia seconda risulta apposto ad alcuni capitoli rimasti inediti cui Cattaneo attese certamente nel periodo, in quanto rispettivamente datati luglio e agosto 1855 e cioè il capitolo, per la prima volta ora pubblicato, Della sensazione, i capitoli Della Memoria e Dell'imaginazione, già inclusi

37 Ivi, 127.

38 Pubblicato per la prima volta in F.U. Saffiotti, Sulla legittimità di una psicologia delle menti associate, in Atti del IV Congresso internazionale di filosofia (1911), Genova, A.F. Formiggini, 1911, III, 640-646; ora riportato in C. Cattaneo, Psicologia, cit., 287-290. 
da Bobbio nell'edizione del 1960 e inoltre altri frammenti ancor meno organici, anch'essi ora inseriti nella presente edizione ${ }^{39}$. Da segnalare infine un testo ascrivibile per varie ragioni agli anni Cinquanta, rimasto a lungo inedito ${ }^{40}$. Nell'intestazione, di pugno dell'autore, si legge: «Psicologia delle menti associate. Prefazione 2 redaz.».

Con ogni verosimiglianza tale materiale fu pensato dallo scrittore milanese come introduzione a un lavoro più generale che aveva in animo di comporre. Se lo si scorre, non si fatica a trovare concetti tra $i$ più tipici del Cattaneo filosofo, già enunciati in modo programmatico negli studi precedenti e destinati a formare il nucleo delle sue meditazioni più tarde: il tema dello sviluppo delle facoltà, condizionato dal vivere sociale. Il carattere limitato delle sensazioni del selvaggio, chiuso nell'orizzonte di un sistema preclusivo, viziato dalla commistione con elementi tradizionali e immaginari. La genesi delle idee in una impresa collettiva in grado di sintetizzare e di valorizzare ciò che è divisione e separazione. La sostituzione dei sensi naturali con una sorte di sensorio comune arricchito incessantemente dal patrimonio analitico. La potenzialità della mente accresciuta dal vivere sociale, perché sostenuta fin nella sua attività più creativa dalla forza inventiva del pensiero trasmesso e condiviso.

È importante sottolineare che in questo abbozzo, come in altri scritti programmatici, si trova una accezione molto vasta del termine idee così come del termine pensiero, esteso ad ogni attività, l'elemento distintivo dell'uomo rispetto ai bruti, il germe costitutivo delle istituzioni, della storia e del mondo civile. Per l'allievo di Romagnosi, per il lettore e interprete di Vico, al fine di rivelare la natura dell'uomo in tutte le sue espressioni si sarebbero dovute interrogare le lingue e le letterature, le arti, le leggi e le religioni. Nella prefazione inedita di cui parliamo la vastità della prospettiva è evidente: «Questa azione e reazione di più intelligenze è veramente necessaria all'attuazione de' sentimenti morali $\gg^{41}$.

Benché intenzionato a concentrarsi nei suoi studi recuperando il tempo dissipato troppo a lungo per sua stessa ammissione dietro «roba

39 Ivi, 265-286.

40 Pubblicato per la prima volta in M. Fugazza, Filosofia e scienze umane: intorno ad alcuni autografi di Cattaneo, cit., 228-238; ora riportato in C. Cattaneo, Psicologia, cit., 89-107.

41 Ivi, 101. 
frusta e roba altrui $\gg^{42}$, Cattaneo diede tuttavia seguito in modo incompleto al disegno tracciato, di cui tale premessa incompiuta rimase tra le carte. Gli impegni lo traevano in varie direzioni. E poi, quasi sempre accadeva che fosse una sollecitazione esterna a indurlo a dare alle sue meditazioni la forma prediletta del saggio. Così accadde anche nel 1857 quando, in risposta a una richiesta di Zenocrate Cesari direttore della Rivista contemporanea di Torino, egli stese l'articolo Un invito alli amatori della filosofia ${ }^{43}$. Stabilendo una interessante continuità, annotò in una lettera: «Ho scritto alcune pagine di filosofia per la Rivista Contemporanea, nel senso che facevo le prefazioni del Politecnico» ${ }^{44}$. Nel testo, evidentemente come risultato delle riflessioni in corso, troviamo riassunti alcuni dei temi ricorrenti del Cattaneo filosofo: il suggerimento di superare la centralità dello studio dei sistemi del passato, e quindi di andare oltre la sterile contemplazione dell'uomo interiore, oltre le vane diatribe dei metafisici, cercando possibili convergenze nella costruzione di una metodologia generale del sapere.

Se gli studi rinnovati erano chiamati a render conto non più delle stutture mentali dell'individuo astrattamente considerato, ma della varietà delle idee e dei costumi degli uomini nel tempo, nonché della vicenda incessante del progresso e del regresso come cifra caratteristica delle civiltà, psicologia e ideologia tradizionalmente intese apparivano ormai inadeguate ai compiti loro affidati ed erano accomunate dall'esigenza di dare un indirizzo differente alle loro ricerche, come era esplicitamente e nuovamente affermato ${ }^{45}$. La strada era aperta per proporre

42 «Io come scrittore ho sciupato il mio tempo lavorando troppo come giornalista di roba frusta e roba altrui invece di far del mio, che la fatica era forse minore; anzi molta roba mia resta dispersa per entro i pasticci fatti delle cose altrui, sicchè non può nemanco parer mia. Con questo fardello di stracci mi vergogno un poco di comparir di nuovo avanti a un publico che non ha perduto il suo tempo». Lettera a $G$. Strambio del 3 gennaio 1855, in Carteggi di Carlo Cattaneo, serie I: Lettere di Cattaneo, III, cit., 198-199.

43 SF, I, 339-357.

44 Lettera a T. Massarani del 26 maggio 1857 in C. Cattaneo, Epistolario, raccolto e annotato da R. Caddeo, Firenze, Barbèra, 1954, III, 21.

45 «La psicologia e l'ideologia s'affaticano invano a ricercare nella mente solitaria dell'individuo nascente l'origine di tutte le sue idee», mentre «fin dai più incerti albori dell'esperienza, la nostra mente oscilla tra i mutui impulsi delle menti associate», in SF, I, 345. 
almeno in abbozzo un sistema entro cui sia facoltà come la memoria, sia operazioni della mente come l'analisi, sia la scienza nel suo insieme, in quanto più elevata produzione umana, fossero pensabili alla luce dell'associazione delle intelligenze collegata alla genesi stessa del pensiero. E passando dalla psicologia all'ideologia, a partire dalle facoltà e dalle operazioni della mente sarebbe divenuto comprensibile il risultato del processo di formazione e trasformazione delle idee, l'ideologia sociale.

\section{LE CONFERENZE ALL'ISTITUTO}

Il nesso tra il saggio uscito a Torino nel 1857 e le conferenze iniziate due anni dopo venne esplicitato dallo stesso Cattaneo, che rivolgendosi all'Istituto Lombardo dichiarava annunciando la prima lezione: «Amando rendervi qualche conto degli studii a cui le occasioni qui avute mi condussero, vorrei, se permettete, potervi leggere una Memoria che posso commisurare al tempo comunque breve che suppongo mi possiate concedere. Essa è in relazione ad altra che pubblicai, ora è poco più di due anni, nella Rivista Contemporanea di Torino» ${ }^{46}$.

L'intervallo era stato impiegato per ulteriori approfondimenti. Lo prova tra l'altro il fatto che pochi mesi prima, nei fascicoli di aprile e di maggio 1859 del Crepuscolo di Carlo Tenca, Cattaneo aveva fatto uscire due brevi saggi riassuntivi della materia cui stava lavorando, sotto il titolo comune di Frammenti di filosofia civile (1852-1859). Il primo articolo conteneva il compendio di un versante significativo della sua produzione di quegli anni, e cioè il tema del pensiero come principio generatore di ricchezza ${ }^{47}$, solo accennato in poche pagine ma destinato ad essere ripreso in un testo più ampio apparso nel 1861 nella nuova serie del Politecnico ${ }^{48}$. Il secondo era dedicato in breve a un argomento, i

46 Lettera alla Presidenza dell'Istituto Lombardo del 16 agosto 1859, riportata in C. Cattaneo, Psicologia, cit., 109.

47 C. Cattaneo, Frammenti di filosofia civile (1852-1859). Del pensiero come principio di pubblica ricchezza, Il Crepuscolo, a. X, n. 7, 15 aprile 1859, 143-148.

48 C. Cattaneo, Del pensiero come principio d'economia publica, uscito in Politecnico, 1861, X, fasc. LVIII, 402-428; in Id., Scritti economici, a cura di A. Bertolino, Firenze, le Monnier, 1956, III, 337-372. Cfr. anche la riedizione, con prefazione di C.G. Lacaita e postfazione di M. Vitale, Milano, Scheiwiller, 2001. 
sistemi $^{49}$, che come abbiamo visto occupava da tempo i suoi interessi, dal momento che dopo il saggio del 1844 era stato sommariamente trattato in alcune parti delle lezioni al Liceo, e sarebbe divenuto oggetto della seconda lettura all'Istituto.

L'accostamento di un tema economico a un altro più propriamente teorico non stupisce: Cattaneo coltivava - lo si è detto - un'accezione molto estesa del termine pensiero, che gli permetteva di riflettere sui fattori immateriali dello sviluppo, all'interno di una visione complessiva dei meccanismi regolativi del vivere associato e delle leggi del progresso. «Je suis économiste et idéologue de mon métier et je n'ai point de penchant et très peu de bon sens [corr. di: «sens commun»] pour la politique», aveva scritto al cognato Anatole Brenier nel $1858^{50}$ : una definizione efficace di un modo di procedere su piani diversi ma convergenti di elaborazione teorica, di cui l'economia e l'ideologia costituivano versanti importanti. Ne è prova anche il progetto, varato nel 1860 , di una riedizione dei saggi economici ${ }^{51}$, da proporre al pubblico nello stesso momento della ripresa di varie iniziative per ricomparire sulla scena culturale milanese.

Il titolo generale apposto ai due lavori pubblicati nel Crepuscolo attesta una volontà di sistemazione teorica che, chiusa la rivista di Tenca, Cattaneo trasferì in altre sedi come il rinato Politecnico, di cui proprio nell'agosto 1859 firmò il contratto ${ }^{52}$, e appunto le letture all'Istituto Lombardo, inaugurate nello stesso mese per riaffermare una presenza nel prestigioso consesso che, non senza alcuni risvolti contrastati legati in particolare alla mancata assegnazione a lui della segreteria,

49 C. Cattaneo, Frammenti di filosofia civile (1852-1859). I sistemi, Il Crepuscolo, a. X, n. 10, 31 maggio 1859, 202-205. Riportato in Id., Psicologia, cit., 165-172.

50 Lettera ad A. Brenier del 2 agosto 1858. Il passo è trascritto da una delle due minute autografe della lettera, conservate in Raccolte storiche del Comune di Milano, Archivio Carlo Cattaneo, cart. 2, pl. XVIII, n. 12, che presenta varianti rispetto a quanto riportato in C. Cattaneo, Epistolario, III, cit., 69.

51 C. Cattaneo, Memorie di economia publica dal 1833 al 1860, Milano, Libreria di F. Sanvito, 1860, di cui venne realizzato solo il volume I.

52 Sui tentativi di ripresa negli anni Cinquanta del periodico e sull'avvio nel 1859-60 della seconda serie, cfr. M. Fugazza, Carlo Cattaneo. Scienza e società 18501868, Milano, FrancoAngeli, 1989, 51-87; C.G. Lacaita, Dal «Politecnico» di Cattaneo al «Politecnico» di Brioschi, Padania, 1993, a. VII, n. 13, 42-92. 
lo riaccolse tra i suoi membri ${ }^{53}$. Rimase invece inattuata l'ipotesi di un incarico di insegnamento in ambito cittadino, di cui si continuò a parlare anche più tardi, come provano gli accenni a una cattedra di «filosofia sociale» che sarebbe stata prospettata per lo scrittore, stando a quanto si legge in alcune lettere scambiate nel 1861 con Gabriele $\operatorname{Rosa}^{54}$.

Tornando alle conferenze all'Istituto, il contenuto della prima di esse è sintomatico. Nel presentarsi all'adunanza, ridimensionando le ambizioni molto estese dell'abbozzo di prefazione citato, dove il riferimento era alle varie espressioni dell'attività umana, lo studioso decise di tracciare i lineamenti di una Psicologia delle scienze. Il presupposto dichiarato era la convinzione dell'impossibilità di distinguere tra il lavoro intellettuale del singolo e l'apporto proveniente dall'elaborazione delle collettività, particolarmente nello sviluppo del pensiero scientifico. Al cuore del cammino della storia venne piantato dunque il tema della scienza ${ }^{55}$. Parlando all'Istituto, in cui i due rami delle scienze matematiche e naturali e delle scienze cosiddette morali correvano in parallelo componendo il mosaico del sapere, lo sforzo di Cattaneo si concentrò in vista dell'obiettivo di una convergenza tra i presupposti del metodo sperimentale e il procedere delle società nella storia, concependo la scienza come gigantesco apparato, esteso in senso sincronico nelle varie parti del globo e nella diacronia delle successive idee trasmesse nella vicenda delle generazioni.

Dopo le premesse e l'introduzione generale, l'attenzione si sof-

53 Per i rapporti dello scrittore con l'Istituto, cfr. G. Bolognesi, Carlo Cattaneo e l'Istituto Lombardo, in Cattaneo, Milano e la Lombardia, cit., 93-123 e, per l'attività svolta nell'ambito dell'ente, nell'ampia panoramica relativa soprattutto agli anni che precedettero il 1848, F. Della Peruta, Cultura e organizzazione del sapere nella Lombardia dell'Ottocento. L'Istituto Lombardo di Scienze e Lettere dalla fondazione all'unità d'Italia, in L'Istituto Lombardo Accademia di Scienze e Lettere (secoli XIX-XX), I: Storia istituzionale, a cura di A. Robbiati Bianchi, Milano, Istituto Lombardo Accademia di Scienze e Lettere-Libri Scheiwiller, 2007, 3-492, passim.

54 Cfr. C. Cattaneo, Epistolario, III, cit., 464 nota.

55 Si osservi tra l'altro che a distanza di pochi mesi Cattaneo destinò alla nuova serie della sua rivista alcuni suoi testi divulgativi di carattere scientifico, che erano parti delle lezioni di cosmologia, e cioè L'uomo nello spazio, L'uomo nel tempo e L'uomo nell'ordine, stampati in Politecnico, 1860, VIII, fasc. XLVI, 343-365; fasc. XLVII, 453-468; fasc. XLVIII, 596-606; in SF, II, 40-123. 
fermò poi su alcuni concetti chiave: i concetti di sistema, oggetto della conferenza del 1860, di antitesi, discusso dopo un lungo intervallo di tre anni in una lezione tenuta nel 1863, e quindi, nel 1864 e nel 186566, di sensazione e analisi. I titoli stessi sottindendono parentele con gli articoli del primo Politecnico e con le lezioni luganesi: un rapporto già più volte sottolineato, a riprova dell'unitarietà dell'impegno di Cattaneo e del legame tra aspetti diversi della sua esperienza intellettuale. Se le lezioni al Liceo erano un terminus a quo da cui è impossibile prescindere, tra le matrici delle letture all'Istituto non vanno dimenticati infatti i saggi prequarantotteschi prima citati, tra cui il saggio già ricordato su Ferrari. Riprendendo quelle lontane osservazioni, recuperate e aggiornate anche per le finalità dell'insegnamento ticinese, non fu casuale che nella seconda conferenza all'Istituto ${ }^{56}$ l'autore riproponesse il concetto di sistema, giungendo a una distinzione più chiara, rispetto a sedici anni prima, tra sistemi chiusi e aperti, gli uni destinati al declino, gli altri capaci di trasformarsi e progredire grazie a una dinamica vitale.

Nonostante il lungo intervallo, una connessione si evidenziò anche rispetto alla terza conferenza sull'antitesi ${ }^{57}$ del novembre 1863. A partire dall'antitesi come atto col quale uno o più individui, «nello sforzo a negare un'idea», giungono a formularne una nuova e viceversa, nell'appropriarsi di un principio, ne negano «anche inconsciamente» un altro, egli tradusse questa definizione in un processo collettivo e storico, identificando l'antitesi con la rottura di ogni sistema unitario e l'immissione di princìpi, in grado di integrarsi o di sostituirsi a quelli dominanti. Nella successione di sistemi lungo il corso storico, non sempre si poteva stabilire una demarcazione netta. Né poteva intendersi come assoluta la distinzione tra sistemi chiusi e aperti, essendo i primi soggetti a modifiche per innesto, i secondi a minacce di involuzione nel caso del prevalere incontrastato di un principio. E poiché in definitiva nella concezione cattaneana un sistema era tanto più progredito, quanto più in esso gli elementi razionali erano riusciti a imporsi su quelli immaginari e fantastici, il termine di riferimento diventava una volta di

56 C. Cattaneo, Psicologia, cit., 141-155. I rapporti di questa lettura con le corrispondenti parti delle lezioni luganesi sono stati sottolineati anche da Bobbio e da D. Castelnuovo Frigessi. Cfr. quanto riportato da Boneschi ivi, 137-138.

57 Ivi, 179-192. 
più la rivoluzione scientifica europea e il tema della scienza tornava prepotentemente al centro della visione della storia ${ }^{58}$.

La lezione sulla sensazione ${ }^{59}$, anticipata in un appunto rimasto inedito del 1855, che rifletteva sul potenziamento dei sensi offerto dai nuovi strumenti di indagine volti a penetrare i segreti della natura, ebbe luogo nel 1864. Tra la fine del 1865 e il 1866 Cattaneo tenne la conferenza sull'analisi, divisa in due parti ${ }^{60}$. Il concetto centrale vi era inteso nella sua massima estensione, essendo la libera analisi indicata come uno dei più grandi interessi materiali e morali dell'umanità. Non tutte le epoche erano state propizie, non tutte le società ne avevano consentito lo sviluppo. A fervidi periodi di innovazioni e scoperte erano seguite lunghe fasi di inerzia. Ancora molto occorreva adoperarsi dunque per ricercare e favorire «i più intimi secreti della vita scientifica». E dal momento che il filo conduttore tornava ad essere l'itinerario del pensiero dalla sua «iniziale semplicità» alle «più difficili evoluzioni» ${ }^{61}$, la lezione si configurava come ricapitolazione dei principali concetti che l'autore aveva indicato a fondamento della sua visione.

Il progetto teorico che Cattaneo si era proposto di realizzare si arrestò qui, dato che la seconda conferenza sull'analisi, a chiusura della serie inaugurata nel 1859, fu nel 1866 l'ultima all'Istituto. Molti spunti erano stati offerti, molte intuizioni presentate con uno stile efficace, a volte vertiginosamente condensato, lo stile di chi voleva perimetrare un nuovo territorio, senza aver tempo e possibilità di poterlo più minuta-

58 Si noti che, evidentemente come nuova tappa di una lunga controversia in atto, tale conferenza si attirò strali particolarmente acuminati dagli avversari di parte clericale conservatrice, in particolare da padre Edoardo Ignazio Daldini di Vezia, che aveva già attaccato l'insegnamento filosofico cattaneano nel 1858 dalle pagine del Credente cattolico, e che contro la lettura compose un corposo libello polemico anonimo di 166 pagine, e cioè Pensieri filosofici sopra un discorso del signor dott. Carlo Cattaneo letto nell'Istituto di scienze e lettere in Milano nell'Adunanza del 12 novembre 1863, Milano, Boniardi e Pogliani, 1865. Per la paternità del testo, cfr. N. Bobbio, Una filosofia militante, cit., 171-178.

59 C. Cattaneo, Psicologia, cit., 197-205.

60 Per la genesi e i tempi di svolgimento delle due letture, si veda quanto ricostruito dalla curatrice, ivi, 213-219, nella nota premessa ai testi.

61 Ivi, 246. 
mente coltivare, ma con l'ansia di concorrere a un avanzamento, a un cambio di passo, a una radicale riforma dei saperi.

\section{UNO SGUARDO AL CONTESTO}

Gli anni in cui lo scrittore conduceva questa sua solitaria riflessione conclusiva erano anni di rapide e profonde innovazioni nelle scienze umane in cui, grazie a scoperte di carattere linguistico, etnologico e paleoantropologico, si aprirono vari filoni di indagine e nacquero nuove discipline, delle quali l'evoluzionismo contribuì a ridefinire $i$ paradigmi. Risentì anche Cattaneo di questo fervore di ricerche? Abbastanza marginalmente, si può affermare, ed è tema che occupa da tempo il campo degli studi la possibilità di ascriverlo a precursore del positivismo $^{62}$, quando non dell'evoluzionismo, di cui ebbe a quanto pare un sentore vago e indiretto. Eppure è singolare osservare che le pagine della Psicologia delle menti associate suonano notevolmente efficaci, dato che, per strade proprie e con una originale sintesi tra elementi appresi e intuizioni nuove, lo scrittore sfiorò nella sua meditazione più tarda motivi che erano già stati presenti alla sua mente e che a causa delle trasformazioni in corso riacquistarono attualità.

Il riferimento è in particolare alla questione, centrale nelle lezioni milanesi, delle cause dello sviluppo o della involuzione intellettuale dei popoli, che portava con sé l'altro grave interrogativo sulla possibilità di stabilire una gerarchia di valore tra gli stessi, secondo criteri che le applicazioni delle scienze della natura al mondo sociale sembravano rendere possibili. Il tema aveva origini antiche, ma proprio negli anni Cinquanta e Sessanta dell'Ottocento ottenne una rinnovata attenzione, in una fase di trasformazioni dei saperi e in presenza di scenari collettivi che rendevano cruciale il confronto. Ed è significativo osservare che, pur non offrendo una sintesi compiuta, gli scritti cattaneani contengono considerazioni importanti nei loro presupposti di fondo.

62 In proposito, ancora una volta illuminante quanto osservato in N. Bobbio, Una filosofia militante, cit., 124-138. Sulla problematicità della tesi di una presunta continuità tra il pensiero di Cattaneo e gli esiti della scuola positiva, cfr. tra l'altro G. Armani, Cattaneo e il positivismo criminologico italiano, in Scienza e filosofia nella cultura positivistica, a cura di A. Santucci, Milano, Feltrinelli, 1982, 390-401. 
Mai garantito una volta per tutte, mai riconosciuto come retaggio e privilegio di alcune stirpi a danno di altre, nelle concezioni dell'autore milanese il progresso risulta fondato soprattutto sull'eguaglianza della natura umana. Eguaglianza basata non su dati immutabili, ma sulla sostanziale uniformità dell'accesso al pensiero. Conciliandosi con una visione problematica e complessa del cammino della storia, tutt'altro che garantito e unilineare, il segreto del progresso o della decadenza appare infatti nelle pagine di Cattaneo il cuore del problema, all'incrocio tra le esigenze della scienza, da salvaguardare nella sua autonomia, e della società, da preservare da ogni spinta autoritaria e nemica della libera indagine.

È importante notare che, proprio dalla metà del secolo, la questione dell'intreccio tra classificazione delle etnie e genesi e sviluppo delle culture stava facendosi più complessa. In un clima che favoriva la trasposizione di concetti biologici nell'area del pensiero sociale, innumerevoli stavano diventando le varianti assunte da dottrine volte a introdurre differenze di valore tra i popoli sparsi sulla superficie del globo. In particolare, in età immediatamente predarwiniana, i tentativi più energici furono condotti dalla scuola antropologica statunitense, impegnata a fissare una scala di nobiltà tra le genti e a provare la loro origine separata, il cosiddetto poligenismo, sulla base di osservazioni e misurazioni craniometriche che occuparono il mondo degli scienziati ${ }^{63}$.

Cattaneo ebbe notizia delle nuove dottrine ${ }^{64}$ (di cui condannò

63 Il maggior esponente di questa scuola, Samuel G. Morton, compendiò i risultati delle sue rilevazioni in Crania Americana; or a comparative view of the skulls of various aboriginal Nations of Norh and South America, to which is prefixed an essay on the varieties of the human species, Philadelphia-London, 1839 e Crania Aegyptiaca; or observations on Egyptian ethnography, derived fron anatomy, history and the monuments, Philadelphia-London, 1844.

64 Alla loro divulgazione contribuì soprattutto il volume Types of Mankind, or Ethnological Researches, based upon the ancient monuments, paintings, sculptures and crania of races and upon their natural, geographical, philological, and biblical bistory, illustrated by selections from the inedited papers of S.G. Morton, by J.C. Nott and Ge.R. Gliddon, Philadelphia-London, 1854. A cura degli stessi autori, cfr. anche Indigenous Races of the Earth, by J.C. Nott and Ge.R. Gliddon, Philadelphia-London, 1857. Significativamente, Nott conobbe e contribuì a far conoscere l'Essai sur l'inegalité des races bumaines di J.A. de Gobineau, che apparve in traduzione negli Stati Uniti nel 1856, con un'appendice antropologica aggiunta dallo stesso Nott. 
come presupposto l'anglosassonismo a sfondo razziale per cui provava una decisa avversione teorica), e proprio negli anni delle conferenze all'Istituto Lombardo vi si oppose con toni vibranti, in nome di una dichiarata simpatia e apertura per le indagini sull'uomo fisico, e insieme della convinzione ben salda che esse poco avessero da dire sullo studio delle vicende del pensiero, governate da regole proprie, che era compito degli studiosi investigare. Lo fece per accenni nelle conferenze all'Istituto e più diffusamente in un saggio della nuova serie della sua rivista $^{65}$, in cui tra le molte affermazioni significative, rivolte a condannare «gli interessi e perciò i timori e i pregiudizii che la schiavitù inspira a chi ne ha profitto», si legge: «È vero che codeste progenie settentrionali, nelle ultime generazioni, fecero inaspettati prodigi d'intelligenza; ma ciò prova che non bisogna disperare d'alcuna parte del genere umano, per quanto inveterata nella primordiale ignoranza. Ai detrattori dei Negri noi per converso additeremo la tarda eppur meravigliosa civiltà dei Britanni e dei Teutoni» ${ }^{66}$.

L'argomento romagnosiano dello scambio e dell'innesto come condizione per lo sviluppo della civiltà, la distinzione precocemente elaborata tra affinità linguistica e affinità razziale, l'approfondimento del concetto di sistema aiutarono insomma il direttore del Politecnico a tenersi ben distinto da teorie volte ad ancorare a basi deterministiche le leggi della trasmissione del sapere e delle culture. Non era perlustrando le vie ancora in gran parte inesplorate che portavano alla spiegazione di una discendenza unica o molteplice, che si poteva chiarire il segreto della decadenza delle nazioni, come è detto nella prima conferenza sulla Psicologia delle menti associate, in cui, dopo aver accennato all'ipotesi dell'esistenza in ogni terra di «una diversa specie del genere umano», così Cattaneo proseguiva: «Vorrei piuttosto prescindere da questa ipotesi nel nostro argumento. Piuttosto direi che se con essa si verrebbe assai facilmente a sciogliere il quesito della primitiva disparità d'intelligenza fra i popoli, ancora non si spiegherebbe come una progenie gen-

65 C. Cattaneo, Tipi del genere umano, Politecnico, 1862, XIV, fasc. LXXV, $336-$ 357; in Id., Scritti storici e geografici, a cura di G. Salvemini e E. Sestan, Le Monnier, Firenze, 1957, III, 214-247.

66 Ivi, 244 e 246. Per riferimenti più generali agli echi del dibattito in ambito italiano, cfr. L'uomo e gli uomini: scritti di antropologi italiani dell'Ottocento, a cura di S. Puccini, Roma, Cisu, 1991. 
tile e sagace, una progenie per molti secoli gloriosa nelle scienze, possa $\mathrm{ad}$ un tratto ricadere nella più profonda impotenza mentale» ${ }^{67}$.

La diffusione delle teorie di Darwin, personalmente ostile alle farraginose classificazioni poligeniste ${ }^{68}$, portò come si sa a un rapido cambiamento di scenari. Non alla fine dei contrasti, ai quali proprio la divulgazione del darwinismo ad opera di interpreti e seguaci avrebbe dato nuovo alimento, in un contesto in cui vari filoni si stavano aprendo nelle scienze dell'uomo, che l'evoluzionismo stava contribuendo a ridisegnare nelle loro coordinate. La stessa questione delle origini e dell'intreccio tra evoluzione e genesi delle culture sarebbe diventata più complessa, nelle dinamiche prodotte dai contatti crescenti tra differenti etnie sparse sulla superficie del globo ${ }^{69}$.

Probabilmente inconsapevole degli effetti dirompenti di dottrine di cui aveva intravisto solo gli albori, Cattaneo, scomparso nel 1869, non ebbe modo di partecipare alle successive controversie, che lo avrebbero avuto in una posizione, una volta di più, originale e propria, dato che, distinguendosi dalle posizioni religioso-spiritualistiche, egli si schierò sino alla fine a difesa dei diritti della scienza nelle indagini sull'uomo $^{70}$. Ma, fautore della libera espressione della mente, mostrò al tempo stesso di respingere ogni tentativo di ricondurre la dimensione

67 C. Cattaneo, Psicologia, cit., 117-118. Vale la pena di notare che, a distanza di alcuni decenni questi motivi furono ripresi nell'ultimo scorcio del secolo da Gabriele Rosa e Arcangelo Ghisleri in polemica con Giovanni Bovio, in merito all'impiego di dottrine antropologiche utilizzate a supporto della politica coloniale. Cfr. A. Ghisleri, Le razze umane e il diritto nella questione coloniale, con l'aggiunta di un capitolo sui "Negri agli Stati Uniti”, Bergamo, Istituto d'arti grafiche, 1896 ( $2^{\mathrm{a}} \mathrm{ed}$. ampliata di una prima versione uscita presso Tipografia Miralta, Savona, 1888).

68 Circa le posizioni fortemente critiche sulla scuola mortoniana espresse dal grande naturalista, cfr. A. Desmond-J. Moore, La sacra causa di Darwin. Lotta alla schiavitù e difesa dell'evoluzione, Milano, Raffaello Cortina Editore, 2012 (ed. originale: Darwin's Sacred Cause, Penguin Books, 2009), su cui cfr. le considerazioni contenute in G. GiorelloT. Pievani, La parte degli angeli. Premessa all'edizione italiana, ivi, IX-XVI.

69 Nell'estesa panoramica di studi, cfr. tra l'altro G.W. Stocking jr., Antropologia dell'età vittoriana, Roma, Ei Editori, 1999 (ed. originale: Victorian Anthropology, New York, The Free Press, 1987).

70 Per un tentativo di inserimento del pensiero del milanese negli svolgimenti della cultura etno-antropologica del suo tempo, cfr. tra l'altro F. Pullè, Proemio a C. Cattaneo, Scritti politici ed epistolario, pubblicati da G. Rosa e J. White Mario, Firenze, Barbèra, 1901, III, pp. XI-XLIII. Sui rapporti con il darwinismo, e più in generale 
intellettuale al piano della costituzione fisica, come efficacemente ebbe a riassumere proprio nell'ultima lezione all'Istituto, sostenendo che l'unità primordiale non si sarebbe dovuta cercare «tra le reliquie del mondo fossile», perché «da dovunque egli sia venuto il genere umano procede alla libera unità del pensiero» ${ }^{71}$.

La seconda delle due lezioni sull'analisi, l'ultima della serie, ebbe luogo come si è ricordato nell'agosto del 1866, oltre due anni dopo la conferenza tenuta da Filippo De Filippi a Torino ${ }^{72}$, che segnò anche in Italia l'inizio delle controversie più accese sul portato delle teorie darwiniane. La Psicologia delle menti associate rimase dunque affidata alle lezioni, che per i limiti di tempo, le caratteristiche dell'ingegno dell'autore e le circostanze della sua vita non riuscirono a convergere in un risultato più compiuto, come Cattaneo stesso non mancò di rilevare amaramente ${ }^{73}$. Durante l'esistenza dello scrittore, le conferenze apparvero in forma compendiata come articoli o estratti negli Atti e nei Rendiconti dell'Istituto ${ }^{74}$. Dopo la sua morte cominciò uno studio più approfondito reso possibile dall'accesso ai manoscritti da parte dei curatori delle prime raccolte, promosse dalla cerchia dei più diretti seguaci ${ }^{75}$, e via via nelle successive edizioni. In diverse stagioni della cultura, i testi delle conferenze milanesi avrebbero poi dato luogo a una serie di interpretazioni, nelle quali Cattaneo sarebbe stato variamente collocato rispetto agli svolgimenti della ricerca filosofica in Italia, dai primi tentativi di sistemazione del suo pensiero ${ }^{76}$, alle riletture degli inizi del Novecento $^{77}$, alle osservazioni gentiliane ${ }^{78}$, ai nuovi studi avviati

sugli echi delle teorie dello scienziato inglese nel Politecnico cattaneano, cfr. M. Fugazza, Carlo Cattaneo. Scienza e società, cit., 127-181.

71 C. Cattaneo, Psicologia, cit., 254.

72 Il testo della celebre comunicazione, intitolata L'uomo e le scimie, venne pubblicato in Politecnico, 1864, XXI , fasc. XCIIII, 5-32, ma da tempo l'antico direttore non aveva più alcuna responsabilità nel periodico.

73 Cfr. la lettera a G. De Castro del 29 novembre 1866, in C. Cattaneo, Epistolario, 1956, IV, cit., 435.

74 Per una esaustiva ricognizione delle diverse versioni e per la loro comparazione, cfr. la Mappatura dei testi in C. Cattaneo, Psicologia, cit., 73-76.

75 C. Cattaneo, Opere edite ed inedite, raccolte da A. Bertani, 7 voll., Firenze, Le Monnier, 1881-1892. I voll. VI e VII, di argomento filosofico, vennero affidati in un primo tempo ad Alberto Mario e, dopo la morte di questi, a Niccola Mameli.

76 Cfr. in particolare G. Cantoni, Il sistema filosofico di Carlo Cattaneo, Rivista 
nel secondo dopoguerra e accompagnati da una sempre più ricca letteratura critica ${ }^{79}$.

Ricostruire tale vicenda è compito che eccede i limiti della presente ricostruzione, a conclusione della quale sembra comunque di poter affermare che, come dimostra la recente pubblicazione promossa dall'Istituto Lombardo, uno dei modi più efficaci per accostarsi oggi a Cattaneo è proprio dar conto del "cantiere" della sua produzione, in cui appunti, progetti, elaborazioni incomplete, quelli che egli stesso definì in più occasioni «frammenti» ${ }^{80}$, offrono prospettive utili per rivisitare il suo itinerario intellettuale. Come in altre parti della produzione

di filosofia scientifica, 1887, s. II, VI, 193-205 e A. Mario, Prefazione, in C. Cattaneo, Opere edite ed inedite, VI, Scritti di filosofia, I, Firenze, Le Monnier, 1892, 5-70.

77 Tra le altre, F. Poggi, Di Carlo Cattaneo filosofo e in particolare della sua psicologia delle menti associate, Oneglia, Nante, 1903 e F.U. Saffiotti, Sulla legittimità di una psicologia delle menti associate, cit.

78 Espresse come è noto in G. Gentile, La filosofia in Italia dopo il 1850. III, I positivisti. Le origini: Carlo Cattaneo (1801-69), La Critica, 1908, a. VI, 105-124, poi in Le origini della filosofia contemporanea in Italia, Messina, Principato, 1921, II, $1-27$.

79 Per i titoli più recenti, cfr. G. De Liguori, Introduzione a C. Cattaneo, Psicologia delle menti associate, Roma, Editori Riuniti, 2000 e S. Moravia, La filosofia di Carlo Cattaneo, in Carlo Cattaneo: i temi e le sfide, cit., 111-121. Per un'ampia rassegna dell'ormai imponente letteratura cattaneana, cfr. G. Armani, Gli scritti su Carlo Cattaneo. Bibliografia 1836-2001, Lugano, G. Casagrande, 2001 e G. Armani e R. Gobbo, Gli scritti su Carlo Cattaneo. Bibliografia, aggiornamento 2001-2005, prefazione di C.G. Lacaita, Lugano, G. Casagrande, 2008. Per un sintetico inquadramento dell'opera dello scrittore milanese nello svolgimento della cultura italiana della seconda metà del secolo XIX, con riguardo soprattutto agli sviluppi della psicologia, cfr. inoltre P. Di Giovanni, Carlo Cattaneo: filosofia civile e psicologia delle menti associate, in Id., Filosofia e psicologia nel positivismo italiano, Roma-Bari, Laterza, 2003, 41-55 e Id., Dalla filosofia alla psicologia nell'Italia post-unitaria, in La nascita delle "scienze umane" nell'Italia post-unitaria, a cura di G. Cimino e G.P. Lombardo, Milano, FrancoAngeli, 2014, 115-127, in particolare 118-121. Cfr. inoltre i riferimenti bibliografici indicati da C.G. Lacaita in C. Cattaneo, Psicologia, cit., 70 nota.

$80 \mathrm{Va}$ osservato che il termine venne recuperato da Arcangelo Ghisleri per la serie da lui promossa degli Scritti completi editi ed inediti di Cattaneo, di cui uscirono solo i primi tre volumi a Milano, presso le Edizioni Risorgimento, tra il 1925 e il 1926. Dopo il primo volume, contenente le Notizie naturali e civili su la Lombardia e saggi sull'agricoltura nell'Alta Italia, il secondo volume raccolse, sotto il titolo Frammenti di 
dello scrittore milanese, anche nelle lezioni all'Istituto Lombardo l'intelaiatura allude a un più vasto disegno rimasto inattuato, le formule sono condensazioni di spunti di cui vorremmo avere una più ampia trattazione, ma le sintesi illuminanti e gli squarci suggestivi della filosofia dell'intelligenza che ne costituisce il fulcro mostrano anche nei loro tratti incompiuti, che offrono stimoli e impediscono facili classificazioni, i caratteri di una perdurante vitalità.

filosofia naturale, parte degli scritti filosofici, tra cui le prefazioni al Politecnico e le lezioni di cosmologia, mentre nel terzo, intitolato Frammenti di filosofia civile, confluì un'altra parte degli scritti filosofici e politici, tra cui la Psicologia delle menti associate. 